\title{
TASK SCHEDULING OF AGV IN FMS USING NON-TRADITIONAL OPTIMIZATION TECHNIQUES
}

\author{
Udhayakumar, P. \& Kumanan, S. \\ Department of Production Engineering, \\ National Institute of Technology, Tiruchirappalli - 620015 , Tamilnadu, India \\ E-Mail: udhaya_kannan@yahoo.com
}

\begin{abstract}
Flexible Manufacturing System (FMS), which is equipped with several CNC machines and Automated Guided Vehicle (AGV) based material handling system is designed and implemented to gain the flexibility and efficiency of production. After the implementation of FMS, in practice, the scheduling of the resources, such as frequent variation in the parts, tools, AGV routings, becomes a complex task. This is being done traditionally using various mathematical programming techniques. In recent years, random search algorithms have been attempted for scheduling. Most of the research has been emphasized only on single objective optimization. Multi objective problems in scheduling with conflicting objectives are more complex and combinatorial in nature and hardly have a unique solution. This paper addresses multi objective task scheduling of AGV in a flexible manufacturing environment using nontraditional optimization algorithms. In this paper the authors made an attempt to find the nearoptimum schedule for two AGVs based on the balanced workload and the minimum traveling time for maximum utilization. The proposed methods are exemplified with illustrations.

(Received in March 2009, accepted in September 2009. This paper was with the authors 3 months for 1 revision.)
\end{abstract}

Key Words: Flexible Manufacturing System, AGV, Task Scheduling, Genetic Algorithm, Ant Colony Optimization Algorithm

\section{INTRODUCTION}

In the present day automated manufacturing environment, flexible manufacturing systems (FMS) are agile and provide wide flexibility. FMS are well suited for simultaneous production of a wide variety of part types in low volumes. FMS is a complex system consisting of elements like workstations, automated storage and retrieval systems, and material handling devices such as robots and AGVs [1]. The FMS elements can operate in an asynchronous manner and the scheduling problems are more complex. Moreover, the components are highly interrelated and in addition, contain multiple part types and alternative routings etc. FMS performance can be enhanced by better co-ordination and scheduling of production machines and material handling equipment [2]. Scheduling is concerned with the allocation of limited resources to tasks overtime and is a decision making process that links the operations, time, cost and overall objectives of the company.

Scheduling of the material handling system in FMS has equal importance as of machines and is to be considered together for the actual evaluation of cycle times. Automated guided vehicles (AGVs) are widely used in flexible manufacturing systems due to their flexibility and compatibility [3]. AGVs can be integrated with the computer controlled production and storage equipment in the shop floor and the entire shop floor operations can be controlled through a computer system. Most of the real world-scheduling problems involve simultaneous optimization of multiple objectives. Dealing with multiple objectives has received much attention over the last few years, where as scheduling is still dominated by the unrealistic single objective approach. The main goal of a multi-objective optimization problem is to 
obtain a set of Pareto optimal solutions that satisfy the constraints [4]. Genetic algorithms (GA) are widely used in the last two decades to address the multi criteria decision problems. Genetic algorithms are non-deterministic stochastic search methods that utilize the theories of evolution and natural selection to solve a problem within a complex solution space [5]. Ant algorithms were proposed by Dorigo et al. [6] as a multi-agent approach to different combinatorial optimization problems.

In this paper the authors have made an attempt to find the near-optimum schedule for two AGVs based on the balanced workload and the minimum traveling time for maximum utilization using Genetic Algorithm and Ant Colony Optimization algorithm.

\section{LITERATURE REVIEW}

Scheduling of FMS has been extensively investigated over the last three decades, and it continues to attract the interest of both the academic and industrial sectors. Various types of scheduling problems are solved in different job shop environments. According to Kustak [7] scheduling in FMS involves scheduling of parts, scheduling of pallets and fixtures, scheduling of tools, and scheduling of material handling system. Several techniques such as mathematical modeling, heuristic based methods and simulation have been applied to study the scheduling and sequencing problems in FMS. Stecke and Morin [8] used mathematical programming and established the optimality of balanced workload for certain types of FMS's. Leung et al. [9] developed an integer-programming model to determine part assignment and tool allocation in a FMS with AGVS. The problem was solved for various numbers of AGVs. Simchi-Levi et al. [10] discussed a sequencing problem to minimize flow time in a network. Algorithms were developed to control a single AGV in a manufacturing system. Sabuncuoglu et al. [11] investigated the performances of machine and AGV scheduling rules against mean flow-time criterion using a simulation model. Karabtik et al. [12] introduced a beam search based algorithm for the simultaneous scheduling of machines and AGVs. Krishnamurthy et al. [13] presented a mixed 0-1 integer-programming model to formulate AGV routing and dispatching problem. The objective of the model is to minimize the total travel time in a network with bidirectional paths. The master problem was decomposed into a set of sub-problems and solved heuristically. Bozer et al. [14] proposed an analytical model to estimate the expected waiting times of loads to be transported. Ganesharajah et al. [15] studied the operational issues of scheduling, dispatching and routing of AGVs in various flow path layouts. Gtindtiz Ulusoyt et al. [16] addressed the problem of simultaneous scheduling of machines and a number of identical automated guided vehicles in a flexible manufacturing system so as to minimize the makespan. Koo et al. [17] presented stochastic travel time models for AGVs to determine loaded and empty vehicle travel times. Van der Meer [18] shows that increasing the capacity of a vehicle results in a reduction of the average throughput time. However, in literature, oneload-carrying AGVs are mainly discussed. Farling et al. [19] recommended the use of the traditional configuration in small systems while using tandem/loop in large systems. Yu et al. [20] proposed partitioning algorithm based on a variable path tandem layout. Qiu et al. [21] presented a scheme to schedule and route a batch of AGVs concurrently on a bidirectional linear path layout. Qiu et al. [22] address the operational issues of scheduling and routing of AGVs. Kim et al. [23] proposed another model for designing tandem systems based on the idea of multi-load vehicles. Levitin et al. [24] developed a routing algorithm when the loads must be retrieved in LIFO fashion. Scheduling and dispatching AGVs in manufacturing systems has been the subject of significant research since the mid-1990s, with various heuristic dispatching rules developed for single-load AGVs (e.g., Veeravalli et al. [25], Abdelmaguid et al. [26], De Koster et al. [27]). Srinivasan Rajagopalan et al. [28] tackled the problem of simultaneously locating the pick-up / drop-off $(\mathrm{p} / \mathrm{d})$ points along the periphery of a 
cell and determining the flowpath for an AGV based material handling system.

The ACO algorithm performs better in problems such as traveling salesperson problem [29], the quadratic assignment problem [30], job shop scheduling problem [31] and the vehicle routing problem [32]. Rajendran et al. [33] developed two ant-colony optimization algorithms and analyzed for solving the permutation flow shop scheduling problem. Blum et al. [34] proposed Max-Min ant system in the hyper-cube framework approach to tackle the broad class of group shop scheduling problem instances. In previous years, most research concerning the AGV scheduling has been focused on developing scheduling algorithms for a single objective such as minimization of the setup costs, minimizing the loading / unloading time. In this paper the authors consider multi objective functions for scheduling the AGV to utilize it to the maximum level.

\section{PROBLEM DESCRIPTIONS}

The type and the operations of FMS differ with configuration. Since the general configuration is not possible, most of the research focuses on specific manufacturing systems. The configuration of the system, the assumptions and the objective criteria in this work are presented in the following sections.

1. In a FMS, AGVs are used to perform the tasks for loading / unloading the parts between the workstations.

2. The assumptions made in this work are as follows:

- The number of AGVs is taken as two.

- The traveling times of AGVs to workstations are considered.

- The AGVs performing the tasks in the workstations are restricted to certain number of times.

- An AGV will not take part in the execution of another task, until it finished the present task assigned i.e. Non pre-emptive scheduling.

- If two AGVs want to enter the same workstation, it is called task collision and permits the AGV, which arrives first to the workstation. If two AGVs arrive simultaneously at the same workstation, AGV having the higher priority is permitted (the priority ordering of AGV is AGV0, AGV1).

3. The objective is to find the near-optimum schedule for two AGVs based on the balanced workload and the minimum traveling time for maximum utilization using Genetic Algorithm (GA) and Ant Colony Optimization (ACO) algorithm. The objective function, which can be defined mathematically for the problem, can be described as follows:

$$
\operatorname{Min} Z=W 1\left(\sum A-\sum B\right)+W 2\left(\sum A+\sum B\right)
$$

$$
\begin{array}{ll}
\text { where: } & \sum A-\text { Sum of the traveling times that are assigned to AGV1 } \\
& \sum B-\text { Sum of the traveling times that are assigned to AGV2 } \\
& W 1, W 2-\text { Weightage factors. }
\end{array}
$$

\section{PROPOSED METHODOLOGIES}

\subsection{Genetic algorithm}

GA is a search algorithm based on the mechanics of the natural selection process (biological evolution). The most basic concept is that the strong tends to adapt and survive while the weak tends to die out. That is, optimization is based on evolution, and the survival of the fittest concept. GAs has the ability to create an initial population of feasible solutions, and then recombine them in a way to guide their search to only the most promising areas of the 
state space. Each feasible solution is encoded as a chromosome (string) also called a genotype, and each chromosome is given a measure of fitness via a fitness (evaluation or objective) function. The fitness of a chromosome determines its ability to survive and produce offspring. A finite population of chromosomes is maintained. GAs use probabilistic rules to evolve a population from one generation to the next. The generations of the new genetic recombination operators such as biased reproduction, crossover and mutation develop solutions. The AGV task scheduling using GA is shown as flowchart in Fig. 1.

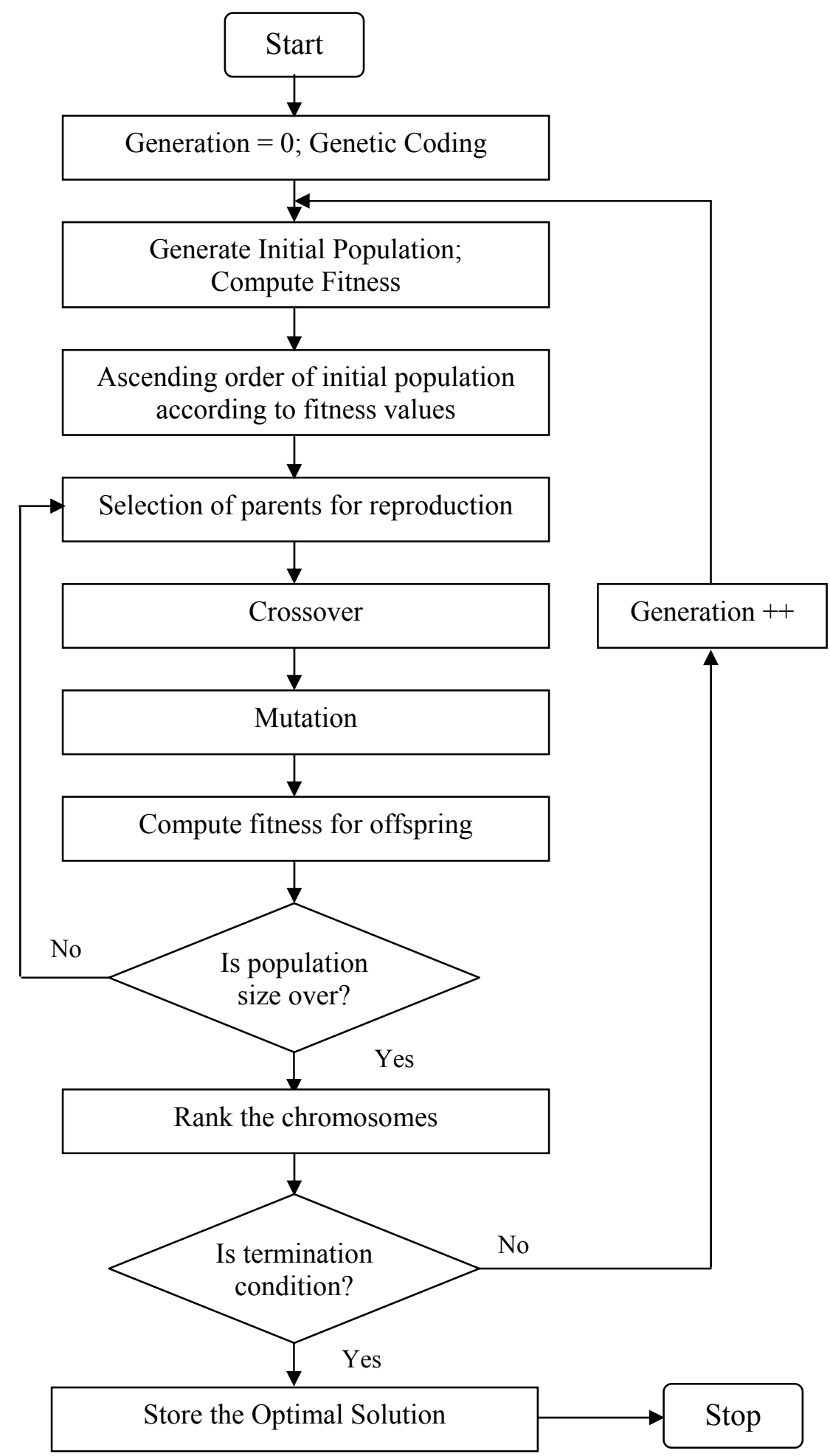

Figure 1: Flowchart for representing the task scheduling of AGV using GA. 
An initial population is generated randomly and the population size is set as $n$. Hence $n$ strings will be created randomly. The chromosome should be in such a way that it contains the information about the solution which it represents. Each bit in the string should represent some characteristic of the solution. In the problem, representation of the AGVs that are performing the tasks for the workstations is given in Fig. 2.

\begin{tabular}{|l|l|l|l|l|l|l|l|l|l|l|l|l|l|l|}
\hline 0 & 3 & 5 & 2 & 0 & 5 & 2 & 1 & 2 & 4 & 5 & 1 & 4 & 3 & 1 \\
\hline
\end{tabular}

Figure 2: Coding of the string.

Here: 0 - Traveling time for AGV1 to perform task 1

1 - Traveling time for AGV2 to perform task 1

2 - Traveling time for AGV1 to perform task 2

3 - Traveling time for AGV2 to perform task 2

4 - Traveling time for AGV1 to perform task 3

5 - Traveling time for AGV2 to perform task 3

The objective function of the problem is to balance the AGVs based on the traveling times assigned to the AGVs and to find the minimum time for the AGVs to perform the tasks that are assigned to it.

Since it is a minimization problem, the objective function value itself is used as fitness function. For obtaining the combined objective function, the fitness values of each objective function is multiplied by some weightage factors and are summed up.

$$
f i t(c)=W 1\left(\sum A-\sum B\right)+W 2\left(\sum A+\sum B\right)
$$

In this paper, the rank selection method is considered to select the chromosomes for reproduction. In this method, first the ranking of the population will be performed. This leads to every chromosome receives fitness from this ranking. The selected strings will undergo crossover and mutation.

The idea behind crossover is that the new chromosome may be better than both of the parents if it takes the best characteristics from each of the parents. Crossover partially exchanges the information between the two selected strings. Each string in the reproduction population is subjected to crossover operation with a specified probability of cross over. The probability of crossover is usually high and can be in the range of 0.6 to 0.9 . In the problem considered, the crossover probability is taken as 0.8 . Once the string is chosen for crossover, its mate and crossover site are selected randomly. The process of cross over is shown in Fig. 3.

\begin{tabular}{|l|l|l|l|l|l|l|l|l|l|l|l|l|l|l|l|}
\hline P1 & 0 & 3 & 1 & 2 & 4 & 3 & 5 & 4 & $\mathbf{1}$ & $\mathbf{4}$ & $\mathbf{0}$ & $\mathbf{5}$ & $\mathbf{0}$ & $\mathbf{3}$ & $\mathbf{2}$ \\
\hline P2 & 1 & 3 & 0 & 3 & 2 & 4 & 4 & 5 & $\mathbf{0}$ & $\mathbf{5}$ & $\mathbf{1}$ & $\mathbf{4}$ & $\mathbf{1}$ & $\mathbf{2}$ & $\mathbf{3}$ \\
\hline
\end{tabular}

\begin{tabular}{|l|l|l|l|l|l|l|l|l|l|l|l|l|l|l|l|}
\hline O1 & 0 & 3 & 1 & 2 & 4 & 3 & 5 & 4 & $\mathbf{0}$ & $\mathbf{5}$ & $\mathbf{1}$ & $\mathbf{4}$ & $\mathbf{1}$ & $\mathbf{2}$ & $\mathbf{3}$ \\
\hline $\mathbf{O} 2$ & 1 & 3 & 0 & 3 & 2 & 4 & 4 & 5 & $\mathbf{1}$ & $\mathbf{4}$ & $\mathbf{0}$ & $\mathbf{5}$ & $\mathbf{0}$ & $\mathbf{3}$ & $\mathbf{2}$ \\
\hline
\end{tabular}

Figure 3: Crossover of the proposed string.

Mutation is the process of randomly modifying the string with a low probability. The purpose of mutation is to introduce new genetic material, or to recreate good genes that were lost by chance through a poor selection of mates. To do this effectively, the effect of mutation must be a major one. At the same time, the valuable gene pool must be protected from wanton 
destruction. Thus, the probability of mutation would be small. The strings, after the crossover operation, are subjected to mutation with 0.05 probability of mutation. Here two sites are selected randomly in a parent string and are mutated. As a result, new offspring is created in which the genes in that two sites are interchanged their positions. The mutation operation is shown in Fig. 4.

\begin{tabular}{|l|l|l|l|l|l|l|l|l|l|l|l|l|l|l|l|}
\hline P1 & 0 & 3 & 5 & 2 & 0 & 5 & 2 & 5 & 2 & 4 & $\mathbf{1}$ & 3 & 1 & 5 & 4 \\
\hline
\end{tabular}

\begin{tabular}{|l|l|l|l|l|l|l|l|l|l|l|l|l|l|l|l|}
\hline $\mathbf{O 1}$ & 0 & 3 & $\mathbf{1}$ & 2 & 0 & 5 & 2 & 5 & 2 & 4 & 5 & 3 & 1 & 5 & 4 \\
\hline
\end{tabular}

Figure 4: Mutation of the proposed string.

After the mutation is completed, the strings are arranged in the ascending order of fitness value. After sorting the strings, the new population is cut down to the old population. Thus, one generation of the genetic process has been completed. This process continues till termination criteria are 100 numbers of generations or a satisfactory pre-defined value for $\mathrm{COF}$, whichever occurs first. The string having the suitable fitness value satisfying both the objective functions is taken from the population and this gives nearer to optimum schedule for the combined objective function.

\subsection{ACO approach}

In this work, the scheduling of the tasks to AGVs in FMS is done using ACO algorithm. The ACO is used to find the combined near-optimum schedule in which it satisfies both the balancing of tasks among the AGVs based on the traveling times and minimized time schedule of the AGVs in an FMS. The proposed ACO approach works in five phases to find the combined objective near-optimum schedule and is explained in Fig. 5. These phases are:

- The initialization phase

- Probabilistic transition rule

- Local trail updating by each ant

- Performance evaluation of each ant

- Global trail updating by a few best ants

ACO approach for the task scheduling of AGVs is as follows: $m$ ants are initially generated and assigned the work stations (WS) to the AGVs. Each ant randomly selects a WS and assigns to an AGV by repeatedly applying the probabilistic transition rule. Ants are guided by the existing pheromone information for the WS-AGV combination. While assigning the WS to an AGV, an ant also modifies the amount of pheromone on the visited edges by applying the local updating rule. Once all workstations are assigned, applying the global updating rule modifies the amount of pheromone for each WS-AGV combination. The global pheromone-updating rule is designed so that it tends to give more pheromone to WSAGV combination, which gets higher reinforcement and raises the probability of selecting the same WS-AGV combination by the ants in the succeeding iterations.

The initial pheromone trail $\left(\tau_{i j}\right)$ for all the WS-AGV combination is kept a constant value. In this proposed method, the initial pheromone trail $\tau_{i j}$ is kept as 0.1 . The number of ants should also be considered and is kept as 20 . 


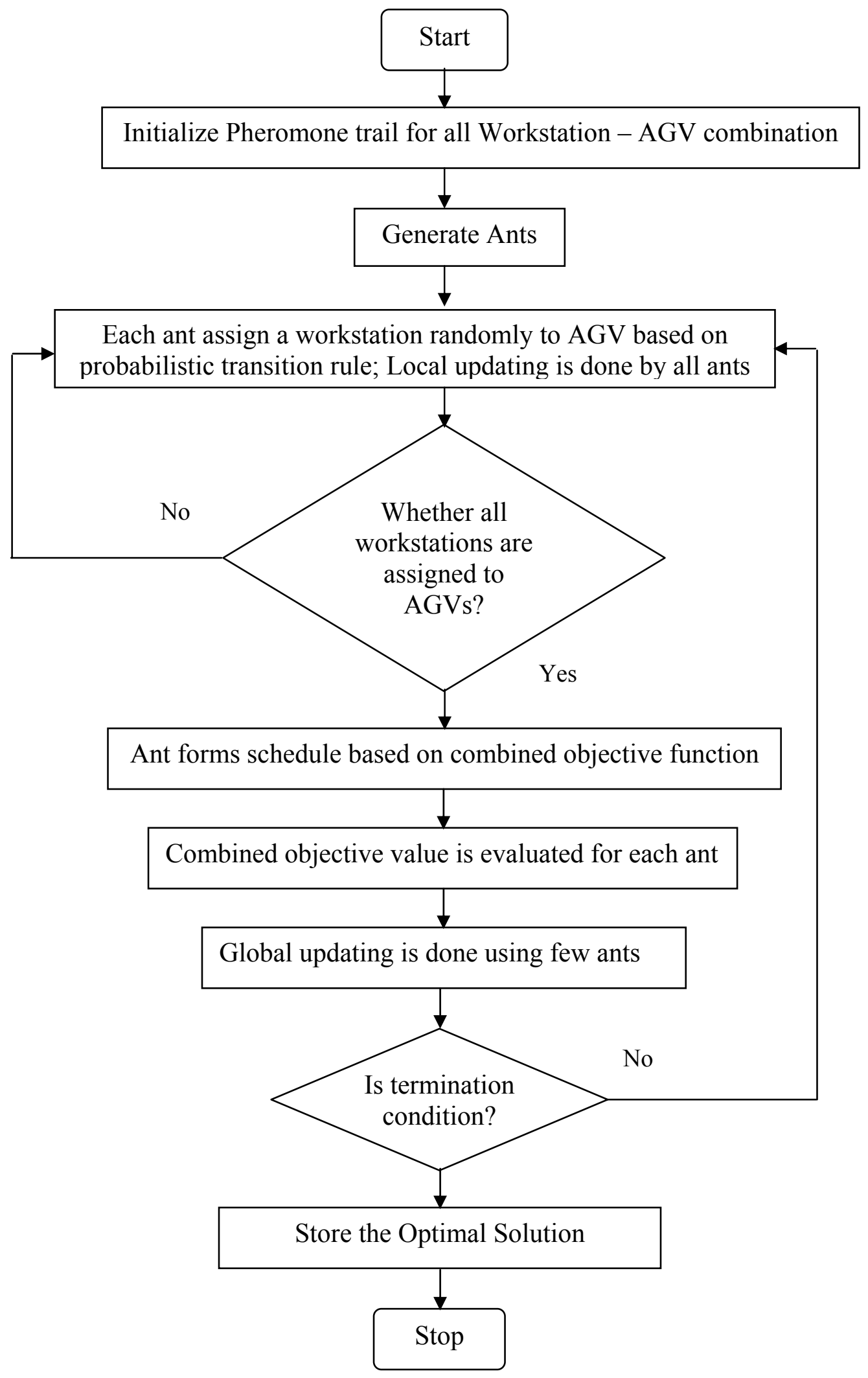

Figure 5: Flowchart for representing the task scheduling of AGV using ACO.

Ants follow a probabilistic transition rule to assign the workstations to AGVs. Ants assign the workstations to AGVs based on the pheromone trail. The ant assigns the workstation to an 
AGV with which it has higher pheromone. The probabilistic transition rule can be represented as:

$$
p_{i j}{ }^{k}=\frac{\tau_{i j}}{\sum_{k=1}^{n} \tau_{i j}}
$$

$p_{i j}{ }^{k}-$ probability that ant $k$ will assign workstation $i$ to $\mathrm{AGV} j$

$n$ - number of AGVs

$\tau_{i j} \quad$ - amount of virtual pheromone between workstation $i$ and $\mathrm{AGV} j$

Initially a constant pheromone trail is kept for each WS-AGV combination. Then ant selects any workstation randomly and assigns to the AGV. Ants have a memory to record the workstations already assigned to AGVs. Ants assign only those workstations to the AGVs, which is not in its memory. Suppose, the probability for any WS-AGV combination is similar to other WS-AGV combination then the ant selects the first higher probability value.

For ant 1 ,

$$
\tau_{31}=0.1, \tau_{32}=0.1
$$

Probability that ant 1 assigns workstation 3 to AGV1

$$
p_{31}{ }^{1}=0.1 /(0.1+0.1)=0.5
$$

Probability that ant 1 assigns workstation 3 to AGV2

$$
p_{32}{ }^{1}=0.1 /(0.1+0.1)=0.5
$$

Since the probability is the same for AGV1 and AGV2, the ant assigns Workstation 3 to AGV1. Similarly the ants will assign the workstations to AGVs based on the probabilistic transition rule.

While building a solution, ants select a workstation, assign to an AGV, and change the WS-AGV pheromone level by applying the local updating rule. The local pheromoneupdating rule is given as per the following equation

$$
\tau_{i j}(t)=(1-\varphi)^{n} \tau_{i j}(t)
$$

where $0 \leq \varphi \leq 1$,

$n$ - number of ants assigned the workstation $i$ to $\mathrm{AGV} j$

$\varphi-$ trail evaporation parameter

After all the ants assign the workstations to AGVs based on the probabilistic rule the pheromone trail for the WS-AGV combination is updated locally using the Eq. (3). If any workstation is assigned to same AGV by more than one ant, then the pheromone trail will be changed based on the number of ants.

$$
\tau_{i j}(t)=(1-\varphi)^{n} \tau_{i j}(t)
$$

where $0 \leq \varphi \leq 1, \quad \varphi=0.3$

For example, Ants 2, 8 and 15 assign workstation 3 to AGV1

$$
\tau_{31}=(1-0.3)^{3} \times 0.1=0.0343
$$

Ants 4, 7, 12, 14 and 17 assign workstation 2 to AGV1

$$
\tau_{21}=(1-0.3)^{5} \times 0.1=0.0168
$$

Normally Local trail updating reduces the trail between WS-AGV combinations and thus reduces the probability of selecting the same combination by the other ants in the same iteration. After local updating the combined objective function is included and the combined objective value is evaluated for each ant.

The global pheromone-updating rule gives more pheromone to AGV-WS combination, which gives higher reinforcement and raises the probability of selecting the same AGV-WS by the ants in the succeeding iterations. 


$$
\tau_{i j}(t+1)=(1-\rho) \tau_{i j}(t)+\sum_{k=1}^{n} \Delta \tau_{i j}(t)
$$

where $\Delta \tau_{i j}(t)= \begin{cases}\frac{1}{C_{0}^{K}}, & \text { if ant } K \text { assigns workstation } i \text { to } \mathrm{AGV} j, \\ 0 & , \text { else }\end{cases}$

where $0<\rho<1$ is the trail evaporation parameter, $\tau_{i j}(t)$ - pheromone trail between workstation $i$ and $\mathrm{AGV} j$ at $t^{\text {th }}$ iteration

$C_{o}{ }^{k}-$ minimum objective value for ant $k$,

$n \quad$ - number of global best ants considered for pheromone trail updating.

In this approach a global list is maintained, which stores the WS-AGV combination and the objective value of a few global best ants. The global list is updated in each iteration after the ants build their solution and before the global trail updating is done. The global pheromone updating is done only for those ants that are present in the global list.

In this problem, the initial pheromone trail kept between workstation and AGV combination is 0.1 . The trail evaporation parameter $\varphi$ influences the local search of the ants. A higher value of evaporation factor increases the tendency of the ants for higher exploration in its search space. A lower value of the parameter leads to the convergence of ants at local minima. The trail evaporation parameter is taken as 0.3 . A higher value of the parameter increases the tendency of the ants to converge at sub-optimal results. Hence the global trail evaporation parameter $\rho$ is taken as 0.3 . The number of ants is kept as 20 . The termination criterion for the ACO algorithm considered in this work is by giving 100 numbers of iterations or a satisfactory pre-defined value for COF whichever occurs first.

\section{CASE STUDY}

In this paper, two AGVs are assigned for transporting the parts among the workstations. Suppose each AGV is doing some task and that traveling time represents the task time, the AGV should be scheduled according to the task times. The objective of the paper is to find near-optimum schedule in which the balancing of the AGVs should be satisfied i.e., the workload for the AGVs should be equal, based on the traveling times and also the traveling time for the optimal schedule should be minimum for the AGVs accomplishing a certain task. Any task has to be performed by both AGV1 and AGV2 for 5 times. The weightage factor given to the objective of balancing the AGVs is 0.6 and for finding the schedule of minimum traveling time of AGVs is 0.4 .

Table I: Problem No. 1.

\begin{tabular}{|c|c|c|c|}
\hline \multirow{2}{*}{ AGV } & \multicolumn{3}{|c|}{ Workstation } \\
\cline { 2 - 4 } & 1 & 2 & 3 \\
\hline 1 & 20 & 25 & 40 \\
\hline 2 & 25 & 30 & 45 \\
\hline
\end{tabular}

Table II: Problem No. 2.

\begin{tabular}{|c|c|c|c|c|}
\hline \multirow{2}{*}{ AGV } & \multicolumn{4}{|c|}{ Workstation } \\
\cline { 2 - 5 } & 1 & 2 & 3 & 4 \\
\hline 1 & 18 & 21 & 43 & 48 \\
\hline 2 & 15 & 16 & 42 & 50 \\
\hline
\end{tabular}

Table III: Problem No. 3.

\begin{tabular}{|c|c|c|c|c|c|}
\hline \multirow{2}{*}{ AGV } & \multicolumn{5}{|c|}{ Workstation } \\
\cline { 2 - 6 } & 1 & 2 & 3 & 4 & 5 \\
\hline 1 & 20 & 25 & 35 & 42 & 54 \\
\hline 2 & 25 & 40 & 45 & 50 & 55 \\
\hline
\end{tabular}




\section{RESULTS AND DISCUSSIONS}

By using various non-traditional optimization techniques, different optimal schedules are obtained with combined objective function for the task scheduling of AGV in an FMS. These techniques optimize two contradicting objectives simultaneously. The main focus of the research is to produce a near optimal schedule for the combined objective function. In each iteration the near-optimum solution obtained is stored and the better solution among the entire iteration is considered as the optimal schedule for the two AGVs. The GA and ACO algorithm is coded in the $\mathrm{VC}++$ language. The computing system used is a Pentium IV processor, $2.66 \mathrm{GHz}$ and $512 \mathrm{MB}$ RAM. The results for the case study problem are shown as below.

Table IV: Combined objective function comparisons of proposed heuristics.

\begin{tabular}{|c|c|c|}
\hline \multirow{2}{*}{ Problem No. } & \multicolumn{2}{|c|}{ Combined Objective function } \\
\cline { 2 - 3 } & $\begin{array}{c}\text { Genetic Algorithm } \\
\text { (GA) }\end{array}$ & $\begin{array}{c}\text { Ant Colony Optimization } \\
\text { Algorithm (ACO) }\end{array}$ \\
\hline 1 & 185 & 184 \\
\hline 2 & 210 & 204 \\
\hline 3 & 242 & 238 \\
\hline
\end{tabular}

From the results, it is found that ACO algorithm performs better, because this algorithm gives minimum combined objective function when compared with GA.

\section{CONCLUSIONS}

The present study in focused on the multi-objective task scheduling of AGV in an FMS using genetic and ant colony optimization algorithm. A scheduling procedure is developed for the task scheduling of AGV with the multiple objective of balancing the AGVs and minimizing the task times of AGVs in an FMS. The near-optimum schedules for the combined objective function are obtained by using GA and ACO algorithm and the results are compared. The result obtained by the ACO algorithm is promising and encouraging. Even a small saving in the combined objective function will cause a significant improvement in task scheduling. This leads to increased utilization of AGV and hence the overall efficiency of the system. This research work leads to the conclusion that the procedures developed in this work can be suitably modified to any kind of FMS for its specific configuration, its problem environment and can be applied for optimizing different objectives separately as well as in combination. When there is a variation in the number of workstations and number of AGV's then the optimization procedure will require to be modified accordingly. Further research will be carried out by relaxing some of the assumptions made in this work and to apply the method to wide range of problems. The applicability of the proposed methodologies has considerable potential applications to manufacturing with further refinement in certain aspects, as outlined below.

- The approach could include other hardware elements of the system to make the scheduling task an integrated one.

- Analysis has to be extended for faster convergence with less iteration and to reduce the possibility of getting trapped in local minima. 


\section{REFERENCES}

[1] Blazewicz, J.; Eiselt, H. A.; Finke, G.; Laporte, G.; Weglarz, J. (1991). Scheduling tasks and vehicles in a flexible manufacturing system, International Journal of Flexible Manufacturing Systems, Vol. 4, 5-16

[2] Anwar, M. F.; Nagi, R. (1998). Integrated scheduling of material handling and manufacturing activities for JIT production of complex assemblies, International Journal of Production Research, Vol. 36, No.3, 653-681

[3] Sabuncuoglu, I.; Hommertzheim, D. L. (1992). Experimental investigation of FMS machine and AGV scheduling rules against the mean flow time criterion, International Journal of Production Research, Vol. 30, No. 7, 1617-1635

[4] Gen, M.; Cheng, R. (1997). Genetic algorithms and engineering design, Wiley, New York

[5] Goldberg, D. E. (1989). Genetic algorithms in search, optimization, and machine learning, Addison-Wesley, Boston

[6] Dorigo, M.; Maniezzo, V.; Colorni, A. (1991). Positive feed back as a search strategy, Technical Report 91-016, Dipartimento di Electronica, Politechnico di Milano, Italy

[7] Kustak, A. (1985). Flexible manufacturing systems: a structural approach, International Journal of Production Research, Vol. 23, No. 6, 1057-1073

[8] Stecke, K. E.; Morin, T. L. (1985). The optimality of balancing workload in certain types of FMS, European Journal of Operations Research, Vol. 20, 68-72

[9] Leung, L. C.; Maheshwari, S. K.; Miller, W. A. (1993). Concurrent part assignment and tool allocation in FMS with material handling considerations, International Journal of Production Research, Vol. 31, 117-138

[10] Simchi-Levi, D.; Berman, O. (1991). Minimizing the total flow time of $n$ jobs on a network, IIE Transactions, Vol. 23, 236-243

[11] Sabuncuoglu, I.; Hommertzheim, D. L. (1992). Dynamic dispatching algorithm for scheduling machines and automated guided vehicles in a flexible manufacturing system, International Journal of Production Research, Vol. 30, 1059-1079

[12] Karabtik, S.; Sabuncuoglu Karabtik, I. (1993). A beam search based algorithm for scheduling machines and AGVs in an FMS, Proceedings of the Second Industrial Engineering Research Conference, 308-312

[13] Krishnamurthy. N. N.; Batta, R.; Karwan, M. H. (1993), Developing conflict-free routes for automated guided vehicles, Operations Research, Vol. 41, 1077-1090

[14] Bozer, Y. A.; Cho, M. S.; Srinivasan, M. M. (1994). Expected waiting times in single-device tripbased material handling systems, European Journal of Operational Research, Vol. 75, 200-216

[15] Ganesharajah, T.; Sriskandarajah, C. (1995). Survey of scheduling research in AGV-served manufacturing systems, Advances in Instrumentation and Control, Proceedings of the International Conference and Exhibition, 87-94

[16] Ulusoy, G.; Sivrikaya-Serifoglu, F.; Bilge, U. (1997). A genetic algorithm approach to the simultaneous scheduling of machines and automated guided vehicles, Computers \& Operations Research, Vol. 24, No. 4, 335-351

[17] Koo, P. H.; Jang, J. (2002). Vehicle travel time models for AGV systems under various dispatching rules, The International Journal of Flexible Manufacturing Systems, Vol. 14, 249261

[18] Van der Meer, J. R. (2000). Operational control of internal transport, ERIM Ph.D. Series Research in Management, Vol. 1

[19] Farling, B. E.; Mosier, C. T; Mahmoodi, F. (2001). Analysis of automated guided vehicle configurations in flexible manufacturing systems, International Journal of Production Research, Vol. 39, No. 18, 4239-4260

[20] Yu, W.; Egbelu, P. J. (2001). Design of a variable path tandem layout for automated guided vehicle systems, Journal of Manufacturing Systems, Vol. 20, 305-319

[21] Qiu, L.; Hsu, W. J. (2001). A bi-directional path layout for conflict-free routing of AGVs, International Journal of Production Research, Vol. 39, 2177-2195

[22] Qiu, L.; Hsu, W. J.; Huang, S. Y.; Wang, H. (2002). Scheduling and routing algorithms for AGVs: A survey, International Journal of Production Research, Vol. 40, No. 3, 745-760 
[23] Kim, K. S.; Jae, M. (2003). A design for a tandem AGVS with multi-load AGVs, International Journal of Advanced Manufacturing Technology, Vol. 22, 744-752

[24] Levitin, G.; Abezgaouz, R. (2003). Optimal routing of multiple-load AGV subject to LIFO loading constraints, Computers \& Operations Research, Vol. 30, 397-411

[25] Veeravalli, B.; Rajesh, G.; Viswanadham, N. (2002). Design and analysis of optimal material distribution policies in flexible manufacturing system using a single AGV, International Journal of Production Research, Vol. 40, 2937-2955

[26] Abdelmaguid, T. F.; Nassef, A. O.; Kamal, B.; Hassan, M. F. (2004). A hybrid GA/heuristic approach to the simultaneous scheduling of machines and automated guided vehicles, International Journal of Production Research, Vol. 42, 267-282

[27] De Koster, R.; Le-Anh, T.; Van der Meer, J. R. (2004). Testing and classifying vehicle dispatching rules in three real world settings, Journal of Operations Management, Vol. 22, 369386

[28] Rajagopalan, S.; Heragu, S. S.; Don Taylor, G. (2004). A Lagrangian relaxation approach to solving the integrated pick-up/drop-off point and AGV flowpath design problem, Applied Mathematical Modelling, Vol. 28, No. 8, 735-750

[29] Dorigo, M.; Gambardella, L. M. (1997). Ant colonies for the travelling salesman problem, Bio Systems, Vol. 43, 73-81

[30] Maniezzo, V.; Colorni, A. (1999). The ant systems applied to the quadratic assignment problem, IEEE Transactions Knowledge and Data Engineering, Vol. 11, No. 50, 769-778

[31] Colorni, A.; Dorigo, M.; Maniezzo, V.; Trubian, M. (1994). Ant system for job-shop scheduling, Belgian Journal of Operation Research, Statistics and Computer Science, Vol. 34, 39-53

[32] Bullnheimer, B.; Hartl, R. F.; Strauss, C. (1998). Applying the ant systems to the vehicle routing problem, Proceedings of the Advances and trends in the local search paradigms for optimisation, 109-120

[33] Rajendran, C.; Zeigler, H. (2004). Ant colony algorithms for permutation flowshop scheduling to minimize makespan / total flowtime of jobs, European Journal of Operational Research, Vol. $155,426-438$

[34] Blum, C.; Samples, M. (2004). An ant colony optimization algorithm for shop scheduling problems, Journal of Mathematical Model Algorithms, Vol. 3, 285-308 